Article

\title{
The Role of Paraoxonase 1 (PON1) as An Oxidation Marker in Muscle Improvement after Increased beta- hydroxybutyrate (BHB) in the Blood of Patients with Multiple Sclerosis ${ }^{\dagger}$
}

\author{
Jose Luis Platero ${ }^{1}$, María Mar López-Rodríguez ${ }^{2}$, Jose Joaquin Ceron ${ }^{3}$, Julia Casani-Cubel 4,* and \\ Jose Enrique de la Rubia Orti ${ }^{4}$ \\ 1 Doctoral Degree School, Catholic University of Valencia San Vicente Martir, 46001 Valencia and Spain; \\ joseluisplateroarmero@gmail.com \\ 2 Department of Nursing, Physiotherapy and Medicine, University of Almería, 04120 Almería, Spain; \\ mlr295@ual.es \\ 3 Interdisciplinary Laboratory of Clinical Analysis, Campus of Excellence Mare Nostrum, University of \\ Murcia, 30100 Murcia, Spain; jjceron@um.es \\ 4 Department of Health Science, Catholic University San Vicente Mártir, 46001 Valencia, Spain; \\ joseenrique.delarubi@ucv.es \\ * Correspondence to: juliacasani@mail.ucv.es; Tel.: +34-600-81-79-03 \\ + Presented at the 1st International Electronic Conference on Brain Sciences, 10-25 November 2020; \\ Available online: https://sciforum.net/conference/IECBS.
}

Received: date; Accepted: date; Published: date

\begin{abstract}
Various studies have concluded that metabolic changes related to energy alterations in multiple sclerosis (MS) are generated at a mitochondrial level. These changes cause a loss of muscle mass and lead to high levels of oxidative stress related to low levels of the enzyme Paraoxonase 1 (PON1). Ketone bodies, mainly beta-hydroxybutyrate (BHB), restore this energy change by improving muscular activity. This pilot study was conducted over 4 months with 51 patients diagnosed with MS, who were randomly divided into an intervention group that received a Mediterranean isocaloric diet supplemented with $60 \mathrm{~mL}$ of coconut oil, and a control group that received the same basal diet without coconut oil. Before and after treatment, anthropometric measurements and blood samples were taken, measuring serum PON1 and BHB concentrations to determine the role of PON1 in muscle improvement after increased BHB in the blood in MS patients. A significant increase in PON1 was obtained, associated with an increase in lean mass and BHB. Thus, it was concluded that this enzyme appeared to be a good marker of decreased oxidation status in MS patients showing muscle improvement after increased BHB in the blood.
\end{abstract}

Keywords: multiple sclerosis; oxidative stress; muscle; betahydroxybutyrate; paraoxonase 1.

\section{Introduction}

Multiple sclerosis (MS) is a chronic neurodegenerative disease of an autoimmune nature, which causes high oxidative stress and progressive loss of skeletal muscle [1,2]. It affects around 2.5 million people worldwide and is the most common cause of non-traumatic neurological disability in young adults in the West [3]. There is currently no curative drug treatment, and the treatments used have numerous side effects [4]. This means that non-pharmacological alternatives must be considered that can help and improve symptoms and prognosis of the disease [5].

Among these alternatives, we can highlight those of a nutritional nature. In this sense, following ketogenic diets has shown benefits in patients with Alzheimer's disease, Parkinson's disease or epilepsy [6-10]. This type of diet enhances, after hepatic metabolism, the production of ketone bodies 
in the blood, which represent an alternative energy source for neurons [11]. Among these ketone bodies, beta-hydroxybutyrate (BHB) is outlined as its high levels show improvements in diseases of a neuronal nature [6]. Specifically, in relation to oxidative stress, an increase in its levels in the blood has been linked to a decrease in free radicals, especially in the brain. As a result, BHB improves cognitive function by protecting neurons, increasing neuronal synapses, preventing neurodegeneration and decreasing inflammation by binding to its HCA2 receptor [12,13]. In terms of anthropometric improvements, ketone bodies and especially BHB are involved in mechanisms related to muscle function, being responsible for increased muscle mass which is accompanied by fat mass loss [14].

In relation to anthropometric changes, where there is an increase in muscle mass, an increase in serum levels of the enzyme paraoxonase 1 (PON1) has been described [15]. Paraoxonases (PONs) are a family of antioxidant enzymes with anti-inflammatory properties, consisting of three members: PON1, PON2 and PON3 [16]. PON1 and PON3 are bound to HDL, and PON2 is an intracellular enzyme located in the mitochondria and endoplasmic reticulum. PON1 is a calcium-dependent glycoprotein synthesised in the liver, whose function is to hydrolyse oxidised fatty acids and protect LDL and HDL lipoproteins from the oxidative process $[17,18]$. The antioxidant activity of PON1 is attributed to its activity in lactase, peroxidase and arylesterase. The function of PON1 as peroxidase leads to the neutralisation of fatty acids and the degradation of $\mathrm{H}_{2} \mathrm{O}_{2}$, an oxidising agent produced as a result of oxidative stress. PON1 also exerts its action on macrophages and other inflammatory cells, preventing cellular oxidative stress and blocking cytokine cascades that aggravate the inflammatory process $[17,19]$. In chronic inflammatory processes, such as MS, the levels and activity of PON1 decrease [20]. In this sense, the reduction in its enzymatic activity is related to different factors: high levels of ROS, physical inactivity, obesity, genetic predisposition (polymorphisms of PON1) and medication intake [18,21-23].

Coconut oil is the source of ketone bodies that stands out, which in turn has antioxidant and anti-inflammatory properties described in neurodegenerative pathologies, such as epilepsy and Alzheimer's disease (AD) $[12,24]$. This oil is characterised by being rich in medium-chain fatty acids (MCFAs), the metabolism of which in the liver promotes the high synthesis of these metabolites in the blood [6].

Based on the background described, the aim of the study is to assess the possible role of PON1 as a marker of oxidative status following possible muscle improvement in MS patients by increasing BHB in the blood.

\section{Materials and Methods}

\subsection{Studio Design}

A pilot, analytical, experimental, prospective and quantitative study was carried out.

\subsection{Subjects}

The main MS societies in the Region of Valencia, Spain, were involved in obtaining the population sample and informed their members of the nature of the study. Inclusion and exclusion criteria were applied to the 67 patients interested in participating in the study. The inclusion criteria used were that the patients were over 18 years of age and had not suffered any relapses in the last year. The exclusion criteria were: pregnant or breastfeeding women, patients with heart complications, with short bowel syndrome, with tracheotomy or stoma, with kidney conditions with creatinine levels twice as high as normal, with liver markers increased 3 times above normal values or with chronic liver disease, patients with dementia, with evidence of drug or alcohol abuse, with acromegaly, with polycystic ovary syndrome (PCOS) or with MS that were included in other research with experimental drugs or treatments. After applying these criteria, the final sample of the study was 51 patients. 


\subsection{Statistical Analysis}

The statistical analysis was performed with the SPSS v.23 tool (IBM Corporation, Armonk, NY, USA). The Mann- Whitney U test was used to compare the two groups and the Wilcoxon signedrange test was used to assess changes before and after intake in the intervention. Categorical data were analysed using a chi-square test. A p value below 0.05 was considered significant. Data are presented as mean \pm standard deviation or number of patients and percentage.

\subsection{Procedure}

Once the population sample was obtained, participants were informed in detail of the nature and objectives of the study, as well as of the importance of not varying the prescribed diet to ensure the validity of the conclusions.

In order to corroborate compliance of the individual treatment, the team made weekly phone calls to all participants to follow up the intervention and to collect information related to the emergence of any kind of side effect.

\subsection{Intervention}

The final sample of 51 MS patients was distributed by double-blind randomisation into an intervention group and a control group. The intervention group received an isocaloric diet for 4 months, which was adapted to the individual characteristics of each participant distributed into 5 intakes per day, consisting of breakfast, mid-morning snack, lunch, afternoon snack and dinner. This diet was supplemented with $60 \mathrm{~mL}$ of extra virgin coconut oil, distributed into two 30-mL intakes (one in the morning and one in the afternoon) with the aim of achieving nutritional ketogenesis in the blood. On the other hand, the control group followed the same isocaloric basal diet as the intervention group over the same 4-month period. This diet consisted of the following percentage distribution of the 3 main macronutrients with respect to total caloric value: $20 \%$ protein, $40 \%$ carbohydrates, and $40 \%$ lipids. Lipid composition was different depending on the group, being mostly provided by coconut oil in the intervention group.

\subsection{Measurements}

The following measurements and determinations were carried out just before and after the 4month intervention, under identical conditions and by the same researcher for each patient.

- Anthropometric measurements: The Faulkner method was applied, following the protocol stipulated by the International Society for the Advancement of Kineantropometry (ISAK).

- Blood analysis and markers: All participants had their blood drawn, just before and after the 4 months and at the same time (9 a.m.) on an empty stomach. After collecting the samples, they were centrifuged to separate the serum from the plasma. BHB levels were calculated with a commercial kit (Randox Laboratories, Crumlin, UK) and PON1 activity was measured using 4nitrophenyl acetate. In both cases, an automatic clinical biochemistry analyser was used (Olympus A 400, Tokyo, Japan).

\section{Results}

\subsection{Population Sample}

A sample of 51 patients with MS was analysed, divided into control and intervention groups, whose socio-demographic and clinical characteristics are shown in Table 1. There were no significant differences between the two groups in the variables analysed before the start of the intervention. 
Table 1. Sociodemographic and clinical characteristics of the study population.

\begin{tabular}{|c|c|c|c|c|c|c|c|}
\hline & & \multicolumn{6}{|c|}{ Group } \\
\hline & & \multicolumn{2}{|c|}{$\begin{array}{c}\text { CG } \\
\mathrm{N}=24\end{array}$} & \multicolumn{2}{|c|}{$\begin{array}{c}\text { IG } \\
\mathrm{N}=27\end{array}$} & \multirow[t]{2}{*}{ Chi2 } & \multirow[t]{2}{*}{$p$} \\
\hline & & Frequency & $\%$ & Frequency & $\%$ & & \\
\hline \multirow{2}{*}{ Types of MS } & Relapsing-Remitting & 17 & $70.8 \%$ & 20 & $74.1 \%$ & .067 & .796 \\
\hline & Secondary-Progressive & 7 & $29.2 \%$ & 7 & $25.9 \%$ & & \\
\hline \multirow{3}{*}{ Gender } & Male & 10 & $41.7 \%$ & 5 & $18.5 \%$ & & \\
\hline & Female & 14 & $58.3 \%$ & 22 & $81.5 \%$ & 3.279 & .070 \\
\hline & & Mean & SD & Mean & SD & $\mathrm{Z}$ & $p$ \\
\hline \multicolumn{2}{|l|}{ Age (years) } & 49.83 & 12.42 & 44.56 & 11.27 & -1.558 & .119 \\
\hline \multicolumn{2}{|c|}{ Time since diagnosis (years) } & 14.25 & 8.36 & 11.93 & 9.7 & -1.428 & 153 \\
\hline \multicolumn{2}{|c|}{ Muscle mass (\%) } & 38.38 & 4.15 & 39.39 & 2.88 & -.547 & .584 \\
\hline \multicolumn{2}{|c|}{ BHB (Mmol/L) } & .05 & .02 & 0.06 & .04 & -.932 & .351 \\
\hline \multicolumn{2}{|l|}{ PON1 (UI/L) } & 2.88 & .77 & 2.67 & .62 & -.832 & .405 \\
\hline
\end{tabular}

Z: U of Mann-Whitney; GC: Control Group; GI: Intervention Group; MS: Multiple Sclerosis; SD: Standard Deviation; PON1: Paraoxonase 1; BHB: Beta-hydroxybutyrate.

\subsection{PON1, BHB Levels and Muscle Mass Percentage.}

With regard to the results of the intervention, the following table (Table 2) shows that there were no significant differences in the control group either in the molecules determined in the PON1 and BHB blood samples, or in the anthropometric variable of muscle percentage. However, when analysing the same results for the same variables in the intervention group, significant differences could be observed in all of them after 4 months of intervention. In particular, there was a significant increase in the levels of PON1 and BHB in the blood after the intervention, as well as in muscle percentage that also increased significantly.

Table 2. Comparison between the values obtained pre-test (before the intervention) and post-test (after the intervention) of the variables paraoxonase 1 (PON1), beta-hydroxybutyrate (BHB) and muscle mass percentage in the control group and intervention group.

\begin{tabular}{lccccccccccccc} 
& \multicolumn{4}{c}{ Control Group } & \multicolumn{4}{c}{ Intervention Group } \\
\cline { 2 - 15 } & \multicolumn{3}{c}{ Pre } & \multicolumn{3}{c}{ Post } & \multicolumn{4}{c}{ Pre } & \multicolumn{4}{c}{ Post } \\
& Mean & SD & Mean & SD & Z & p & Mean & SD & Mean & SD & Z & p \\
\hline PON1 (UI/L) & 2.88 & .77 & 2.87 & .82 & -1.586 & .113 & 2.67 & .62 & 2.92 & 0.68 & -3.722 & $.001^{*}$ \\
BHB (Mmol/L) & .05 & .02 & .04 & .04 & -1.254 & .210 & .06 & .04 & .10 & .10 & -2.005 & $.045^{*}$ \\
Muscle Mass (\%) & 38.38 & 4.15 & 37.79 & 4.14 & -1.738 & .082 & 39.39 & 2.88 & 40.22 & 2.86 & -2.955 & $.003^{*}$ \\
\hline
\end{tabular}

Z: Wilcoxon test; BHB: Beta-hydroxybutyrate; PON1: Paraoxonase 1; SD: Standard deviation.

\section{Discussion}

There is scientific evidence on the relationship between diet and reduced symptoms of neurodegenerative diseases such as AD, Parkinson's disease, epilepsy and others [11]. As far as MS is concerned, although it differs from other neurodegenerative diseases in terms of clinical and pathogenic conditions, it shares common processes, with a high level of oxidative stress being particularly evident [3]. This fact, alongside the current lack of therapeutic cure for MS, leads us to consider the intervention with coconut oil as a source of BHB in the blood in this study. Coconut oil has already shown benefits in other neurodegenerative diseases [24-26].

After the intervention, which was based on administering large amounts of medium-chain fatty acids contained in an isocaloric diet supplemented with coconut oil, a significant increase in BHB was observed in the intervention group that did not occur in the control group. In this sense, other published studies indicate that the fats provided by coconut oil have a neuroprotective effect as they 
increase the percentage of BHBs that have anti-inflammatory properties [24,26]. They are also related to an increase in lean mass, which in these patients decreases progressively as the disease progresses $[8,14,26,27]$. Therefore, the results of our study would coincide with those obtained by other authors, in which the production of ketone bodies through the diet was related to improvements in metabolism and motor function [8], since we observed that there was a significant increase in the percentage of muscle in the patients who received the coconut oil that did not occur in the control group. In addition to the satiating effect, the effect of BHB related to anthropometric changes characterised by an increase in muscle mass has also been associated with the ability to restore mitochondrial activity, predictably by a decrease in ROS and RNS $[5,8,28]$.

On the other hand, a significant increase in the levels of PON1 synthesised in the liver is observed in the intervention group. PON1 is a marker associated with low levels of oxidative stress and inflammation [19]. Neuroprotective properties have been attributed to this enzyme, which is an integral part of HDL, as well as an important role in the prevention of atherosclerosis [29]. As regards its location, it has been found in various types of tissue, including skeletal muscle [30]. In this sense, increased levels of PON1 in the blood are in turn related to greater oxygen consumption and greater muscular strength [31] which would explain how it is positively correlated with increased muscle mass in other pathologies [15]. In addition, lower activity of PON1 is linked to the development of neurodegenerative diseases [29]. Therefore, our results could indicate that muscle improvement could be related to significant increases in the enzyme in MS. Thus, we believe that further research should be conducted to investigate the possibility that PON1 may be used as a diagnostic tool to improve the oxidative state of this disease, when motor improvements related to decreased oxidative stress occur.

However, despite the results obtained, we found clear limitations to be taken into account for future trials. As this is a pilot study, the population used in the study has been limited, so we propose to replicate the study with a more representative population. In this sense, it would be interesting to see the evolution of the levels of PON1 and BHB throughout the intervention, and not only before and after the study in order to better understand the behaviour of both molecules and their relationship throughout the intervention.

\section{Conclusions}

Responding directly to the proposed objective, it is concluded that the enzyme PON1 could be related to oxidation status when muscle improvement occurs in MS patients, after an increase of BHB in blood.

Author Contributions: Conceptualisation, J.E.d.1.R.O.; Data curation, J.L.P.; Formal analysis, J.E.d.l.R.O. and M.M.L.-R.; Funding acquisition, J.E.d.l.R.O.; Investigation, J.J.C.; Methodology, M.M.L.-R.; Project administration, J.C.-C.; Resources, J.C.-C.; Software, M.M.L.-R.; Validation, J.J.C.; Writing-original draft, J.L.P.; Writing-review \& editing, J.E.d.1.R.O. All authors have read and agreed to the published version of the manuscript.

Funding: This research was funded by the Catholic University Foundation San Vicente Mártir, for the research project The Impact of Triglycerides on Multiple Sclerosis (Promotion code 2018-203-001).

Acknowledgments: The authors would like to thank all MS patients and the associations for their participation.

Conflicts of Interest: The authors declare no conflict of interest.

\section{References}

1. Racosta, J.M.; Kimpinski, K. Autonomic dysfunction, immune regulation, and multiple sclerosis. Clin. Auton. Res. 2016, 26, 23-31, doi:10.1007/s10286-015-0325-7.

2. Lassmann, H.; van Horssen, J.; Mahad, D. Progressive multiple sclerosis: pathology and pathogenesis. Nat. Rev. Neurol. 2012, 8, 647-656, doi:10.1038/nrneurol.2012.168

3. Dendrou, C.A.; Fugger, L.; Friese, M.A. Immunopathology of multiple sclerosis. Nat. Rev. Immunol. 2015, 15, 545-558, doi:10.1038/nri3871.

4. Tobin, W.O. Management of Multiple Sclerosis Symptoms and Comorbidities. Continuum 2019, 25, 753- 
772, doi:10.1212/CON.0000000000000732

5. Miller, E.D.; Dziedzic, A.; Saluk-Bijak, J.; Bijak, M. A Review of Various Antioxidant Compounds and their Potential Utility as Complementary Therapy in Multiple Sclerosis. Nutrients. 2019, 11, 1528, doi:10.3390/nu11071528.

6. Taylor, M.K.; Swerdlow, R.H.; Sullivan, D.K. Dietary Neuroketotherapeutics for Alzheimer's Disease: An Evidence Update and the Potential Role for Diet Quality. Nutrients 2019, 11, 1910, doi:10.3390/nu11081910.

7. Gumus, H.; Bayram, A.K.; Kardas, F.; Canpolat, M.; Çağlayan, A.O.; Kumandas, S.; Kendirci, M.; Per, H. The Effects of Ketogenic Diet on Seizures, Cognitive Functions, and Other Neurological Disorders in Classical Phenotype of Glucose Transporter 1 Deficiency Syndrome. Neuropediatrics 2015, 46, 313-320, doi:10.1055/s-0035-1558435.

8. Bahr, L.S.; Bock, M.; Liebscher, D.; Bellmann-Strobl, J.; Franz, L.; Prüß, A.; Schumann, D.; Piper, S.K.; Kessler, C.S.; Steckhan, N.; et al. Ketogenic diet and fasting diet as Nutritional Approaches in Multiple Sclerosis (NAMS): protocol of a randomized controlled study. Trials 2020, 21, 3, doi:10.1186/s13063-0193928-9.

9. Ye, F.; Li, X.J.; Jiang, W.L.; Sun, H.B.; Liu, J. Efficacy of and patient compliance with a ketogenic diet in adults with intractable epilepsy: a meta-analysis. J. Clin. Neurol. 2015, 11, 26-31, doi:10.3988/jcn.2015.11.1.26.

10. Klement, R.J.; Champ, C.E.; Otto, C.; Kämmerer, U. Anti-Tumor Effects of Ketogenic Diets in Mice: A MetaAnalysis. PLoS ONE 2016, 11, e0155050, doi:10.1371/journal.pone.0155050.

11. Maalouf, M.; Rho, J.M.; Mattson, M.P. The neuroprotective properties of calorie restriction, the ketogenic diet, and ketone bodies. Brain Res. Rev. 2009, 59, 293-315. doi:10.1016/j.brainresrev.2008.09.002.

12. Stafstrom, C.E.; Rho, J.M. The ketogenic diet as a treatment paradigm for diverse neurological disorders. Front. Pharmacol. 2012, 3, 59, doi:10.3389/fphar.2012.00059.

13. Offermanns, S.; Schwaninger, M. Nutritional or pharmacological activation of HCA(2) ameliorates neuroinflammation. Trends Mol. Med. 2015, 21, 245-255, doi:10.1016/j.molmed.2015.02.002.

14. Mumme, K.; Stonehouse, W. Effects of medium-chain triglycerides on weight loss and body composition: a meta-analysis of randomized controlled trials. J. Acad. Nutr. Diet. 2015, 115, 249-63, doi:10.1016/j.jand.2014.10.022.

15. Vecka, M.; Jáchymová, M.; Vávrová, L.; Kodydková, J.; Macášek, J.; Urbánek, M.; Krechler, T.; Slabý, A.; Dušková, J.; Muravská, A.; et al. Paraoxonase-1 (PON1) status in pancreatic cancer: relation to clinical parameters. Folia Biol. 2012, 58, 231-237.

16. Reichert, C.O.; de Macedo, C.G.; Levy, D.; Sini, B.C.; Monteiro, A.M.; Gidlund, M.; Maselli, L.M.F.; Gualandro, S.F.M.; Bydlowski, S.P. Paraoxonases (PON) 1, 2, and 3 Polymorphisms and PON-1 Activities in Patients with Sickle Cell Disease. Antioxidants 2019, 8, 252, doi:10.3390/antiox8080252.

17. Menini, T.; Gugliucci, A. Paraoxonase 1 in neurological disorders. Redox Rep. 2014, 19, 49-58, doi:10.1179/1351000213Y.0000000071.

18. Costa, L.G.; Giordano, G.; Furlong, C.E. Pharmacological and dietary modulators of paraoxonase 1 (PON1) activity and expression: the hunt goes on. Biochem. Pharmacol. 2011, 81, 337-344, doi:10.1016/j.bcp.2010.11.008.

19. Tisato, V.; Romani, A.; Tavanti, E.; Melloni, E.; Milani, D.; Bonaccorsi, G.; Sanz, J.M.; Gemmati, D.; Passaro, A.; Cervellati, C. Crosstalk Between Adipokines and Paraoxonase 1: A New Potential Axis Linking Oxidative Stress and Inflammation. Antioxidants 2019, 8, 287, doi:10.3390/antiox8080287.

20. Castellazzi, M.; Trentini, A.; Romani, A.; Valacchi, G.; Bellini, T.; Bonaccorsi, G.; Fainardi, E.; Cavicchio, C.; Passaro, A.; Zuliani, G.; et al. Decreased arylesterase activity of paraoxonase-1 (PON-1) might be a common denominator of neuroinflammatory and neurodegenerative diseases. Int. J. Biochem. Cell Biol. 2016, 81 (Pt B), 356-363, doi:10.1016/j.biocel.2016.06.008.

21. Boshtam, M.; Razavi, A.E.; Pourfarzam, M.; Ani, M.; Naderi, G.A.; Basati, G.; Mansourian, M.; Dinani, N.J.; Asgary, S.; Abdi, S. Serum paraoxonase 1 activity is associated with fatty acid composition of high density lipoprotein. Dis. Markers. 2013, 35, 273-280, doi:10.1155/2013/612035.

22. Shunmoogam, N.; Naidoo, P.; Chilton, R. Paraoxonase (PON)-1: a brief overview on genetics, structure, polymorphisms and clinical relevance. Vasc. Health Risk Manag. 2018, 14, 137-143, doi:10.2147/VHRM.S165173.

23. Fridman, O.; Fuchs, A.G.; Porcile, R.; Morales, A.V.; Gariglio, L.O. Paraoxonase: its multiple functions and pharmacological regulation. Arch. Cardiol. Mex. 2011, 81, 251-60.

24. Nafar, F.; Clarke, J.P.; Mearow, K.M. Coconut oil protects cortical neurons from amyloid beta toxicity by 
enhancing signaling of cell survival pathways. Neurochem. Int. 2017, 105, 64-79, doi:10.1016/j.neuint.2017.01.008.

25. Ułamek-Kozioł,, M.; Czuczwar, S.J.; Januszewski, S.; Pluta, R. Ketogenic Diet and Epilepsy. Nutrients 2019, 11, 2510.

26. De la Rubia Ortí, J.E.; García-Pardo, M.P.; Drehmer, E.; Sancho Cantus, D.; Julián Rochina, M.; Aguilar, M.A.; Hu Yang, I. Improvement of Main Cognitive Functions in Patients with Alzheimer's Disease after Treatment with Coconut Oil Enriched Mediterranean Diet: A Pilot Study. J. Alzheimers Dis. 2018, 65, 577587, doi:10.3233/JAD-180184.

27. Veech, R.L. The therapeutic implications of ketone bodies: the effects of ketone bodies in pathological conditions: ketosis, ketogenic diet, redox states, insulin resistance, and mitochondrial metabolism. Prostaglandins Leukot Essent Fatty Acids 2004, 70, 309-319, doi:10.1016/j.plefa.2003.09.007.

28. Veldhorst, M.A.; Westerterp, K.R.; Westerterp-Plantenga, M.S. Gluconeogenesis and protein-induced satiety. Br. J. Nutr. 2012, 107, 595-600, doi:10.1017/S0007114511003254.

29. Levy, D.; Reichert, C.O.; Bydlowski, S.P. Paraoxonases Activities and Polymorphisms in Elderly and OldAge Diseases: An Overview. Antioxidants 2019, 8, 118.

30. Marsillach, J.; Mackness, B.; Mackness, M.; Riu, F.; Beltrán, R.; Joven, J.; Camps, J. Immunohistochemical analysis of paraoxonases-1, 2, and 3 expression in normal mouse tissues. Free Radic Biol. Med. 2008, 45, 146157, doi:10.1016/j.freeradbiomed.2008.03.023.

31. Russo, A.; Pirisinu, I.; Vacca, C.; Reginato, E.; Tomaro, E.S.; Pippi, R.; Aiello, C.; Talesa, V.N.; De Feo, P.; Romani, R. An intensive lifestyle intervention reduces circulating oxidised low-density lipoprotein and increases human paraoxonase activity in obese subjects. Obes. Res. Clin. Pract. 2018, 12 (Suppl 2), 108-114, doi:10.1016/j.orcp.2016.11.006.

Publisher's Note: MDPI stays neutral with regard to jurisdictional claims in published maps and institutional affiliations.

(C) 2020 by the authors. Licensee MDPI, Basel, Switzerland. This article is an open access article distributed under the terms and conditions of the Creative Commons Attribution (CC BY) license (http://creativecommons.org/licenses/by/4.0/). 